\title{
Hallazgo de arteria lusoria en el estudio de otitis media a repetición. A propósito de una observación pediátrica y revista breve de la literatura
}

\author{
Arteria Lusoria revealed by a accute media otitis. \\ About a pediatric observation and brieve review of literatura
}

\author{
K. Ait idir'.
}

\section{RESUMEN}

Arteria Lusoria (AL) o arteria subclavia aberrante derecha es una malformación vascular rara. Es la más común de las malformaciones del arco aórtico. Fue descrita por primera vez por Hunalud en 1735. La etiología no está clarificada hasta hoy en día. AL puede formar parte de un cuadro sindrómico (síndrome de Down, síndrome de Edwards) o de una malformación cardiaca compleja. Es casi siempre asintomática, pero algunas veces puede revelarse por signos respiratorios o disfagia. Se elige habitualmente el tratamiento conservador. Les presentamos el caso de un niño de 2 años que presentó una $A L$ revelada atípicamente por otitis media a repetición.

Palabras clave: Arteria Lusoria, disfagia, otitis media crónica, vascular.

\section{ABSTRACT}

Arteria Lusoria (AL) or aberrant right subclavian artery is a rare congenital aortic arch malformation. Hunauld described it for the first time in 1735. The cause is until now no totally clarified. Usually it is associated with chromosomal disorders such as Down's syndrome, Edwards's syndrome or appears in childhood with complexes cardiovascular abnormalities. Patients are frequently asymptomatic, but it may be revealed by respiratories symptoms or by a dysphagia. The treatment is generally conservative. We expose a case report about a child of 2 years old with AL diagnosed after several episodes of purulent otitis.

Key words: Arteria Lusoria, dysphagia, chronic media otitis, Vascular.

\section{INTRODUCCIÓN}

La arteria subclavia aberrante, 0 el síndrome de la arteria subclavia aberrante 0 arteria subclavia Iusoria $(A L)$ es el término referido a una rara variante anatómica del origen de la arteria subclavia derecha y corresponde a una de las anomalías congénitas vasculares más comunes del arco aórtico.

Su estudio puede ser realizado por una TC de tórax en fase vascular, una angiografía de cuello,

1 Servicio de Pediatría. Centro Especializado Militar en Consultaciones. Hussein-Dey Argel.

Recibido el 28 de agosto, 2016. Aceptado el 1 de octubre, 2016. 
una radiología contrastada de esófago y en un estudio radiológico por deglución.

La AL se inicia distal a la subclavia izquierda cruza detrás del mediastino y del esófago. Puede causar un anillo vascular alrededor de la tráquea y el esófago por lo que es posible tener síntomas de disfagia (denominada disfagia lusoria) o provocar parálisis del recurrente. En este último caso se habla del síndrome de Ortner. La AL puede ser originada de un segmento dilatado desde la aorta descendente.

Cuando la AL es sintomática el tratamiento es quirúrgico y su diagnóstico es habitualmente un hallazgo de estudios de imágenes solicitados por otras causas pero puede tener serias complicaciones si se obtiene en forma tardía.

\section{CASO CLÍNICO}

Se presenta el caso de H. Mohammed Mehdi, varón de 2 años. Es el tercer hijo de una pareja noconsanguínea, sin antecedentes médicos. Presentó una bronquiolitis a 4 meses relacionada con un reflujo gastroesofágico. Luego, manifestó otitis media a repetición con supuración (5 episodios en los 19 primeros meses) tratadas cada vez por antibióticos orales y locales.

El examen clínico encontró un niño eutrófico, afebril, con supuración bilateral de los oídos, de mal olor en el examen físico. Sin otras alteraciones en el examen físico. Ante esta historia y cuadro clínico, se decide practicar un tránsito esofagogastroduodenal que mostraba una huella profunda en el esófago superior evocando una anomalía del arco aórtico y también visualizaba un reflujo gastroesofágico mínimo (Figura 1: A, $B, C)$. Conjuntamente, se realizó una TC contrastada de tórax que mostró un trayecto aberrante de la arteria subclavia derecha cruzando por detrás del esófago y siendo interpretada como AL (Figura 2: D, E, F). Otros estudios como ecografía cardiaca y ecotomografía de abdomen fueron normales. El estudio bacteriológico de la secreción purulenta no reveló gérmenes patógenos.

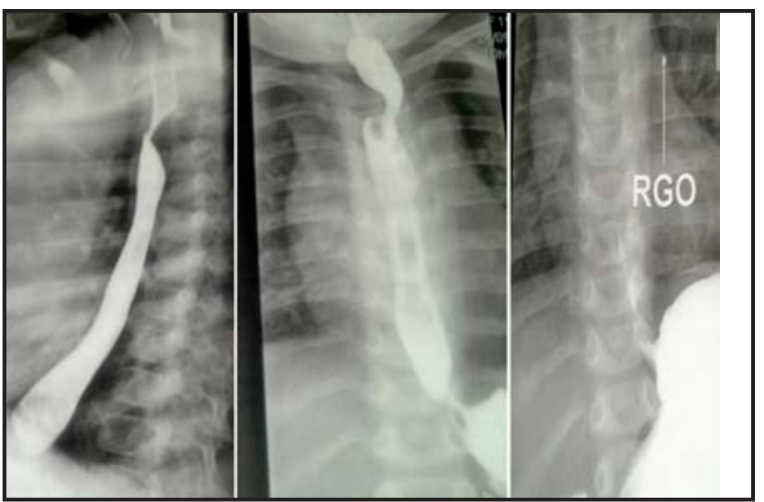

Figura 1. Esofágo vista frontal y la huella de perfil (A, B), la ERGE mínima (C).

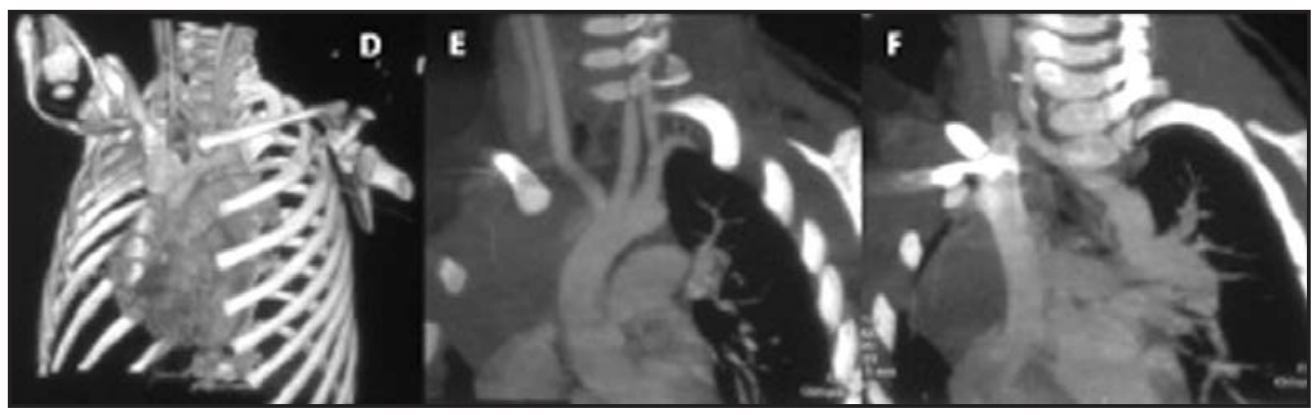

Figura 2. Imagen en 3D (D) del trayecto aberrante de la arteria subclavia derecha cruzando por detrás del esófago. 
La ausencia de síntomas generados por esta anomalía congénita de la arteria subclavia derecha y su pesquisa como un hallazgo radiológico de examen motivó solo una conducta de vigilancia clínica de esta anomalía. Para el reflujo se inició un tratamiento en base a inhibidor de la bomba de protón, durante dos meses. Para su otitis media el tratamiento fue el convencional realizado en estos pacientes. Luego de dos años de observación, Mehdi tiene ahora 4 años, ha presentado una evolución favorable sin síntomas atribuidos a su malformación.

\section{DISCUSIÓN}

Las anomalías de los arcos aórticos son anomalías de origen o de posición de la aorta, de los vasos de la raíz 0 de las arterias pulmonares susceptibles de comprimir el eje tráqueo-digestivo. La arteria subclavia aberrante derecha (ASAD) $0 \mathrm{AL}$ es la anomalía del arco aórtico más común, su prevalencia es estimada entre 0,2\%-2,5\% ${ }^{1}$. La palabra lusoria es de origen latino Iusorius o Iusus naturae significando relativo al juego, queriendo objetivar un variante de la normal2.

La AL fue descrita por primera vez en 1735 por Hunalud seguido por David Bayford en 17493,4, Luego Gross en 1946, Gaujoux en 1968 y Bayford en $1974^{6}$. Todas estas descripciones fueron relacionadas con el contexto de disfagia. La primera descripción radiológica fue realizada en 1936 por Kommrell utilizando un tránsito esofagogastroduodenal ${ }^{7}$. No hay datos sobre el sex-ratio ${ }^{8}$.
Cuatro vasos provienen secuencialmente del arco aórtico: las arterias carótidas comunes derecha e izquierda, la arteria subclavia izquierda (ASI) y la ASAD. Esta última viene de la aorta descendente y cruza hacia arriba y la derecha en el mediastino posterior. El trayecto retroesofágico resulta de la involución o de la ausencia del cuarto arco aórtico derecho con una persistencia de la ASI (Figuras 3A-3B). La ASI puede ser atada a la aorta descendente por un pequeño segmento que puede presentar una dilatación aneurismal conocida por el divertículo de Kommerell'. Una arteria subclavia aberrante izquierda puede ser observada con un arco aórtico derecho realizando una imagen en espejo ${ }^{1}$.

La etiología sigue siendo desconocida, quizás resulta de una disminución del flujo sanguíneo 0 secundaria a anomalías embrionarias con implicación de las células de la cresta neural y de las múltiples vías de señalización ${ }^{9-11}$.

La AL es retroesofágica en $80 \%-83 \%$ de los casos, puede tener un trayecto inter-tráqueo-esofágico en $12 \%-15 \%$ o pretraqueal solo en $4 \%{ }^{10-12}$. Se encuentra más frecuentemente en niños con síndrome de Down (primera citación en 1950), síndrome de Edwards y trisomía 13 sino también en pacientes triploides, monocómicos $\mathrm{X}$ con depleción $22^{13,14}$.

La asociación con cardiopatías congénitas no es excepcional y ocurre en $2,9 \%-4,4 \%$ de los casos. En primer lugar las malformaciones conotroncales $(38 \%)$, seguidas por los defectos septales $(28 \%)$, las patologías obstructivas $(26 \%)$, la tetralogía de Fallot (12\%), coartación de la aorta y

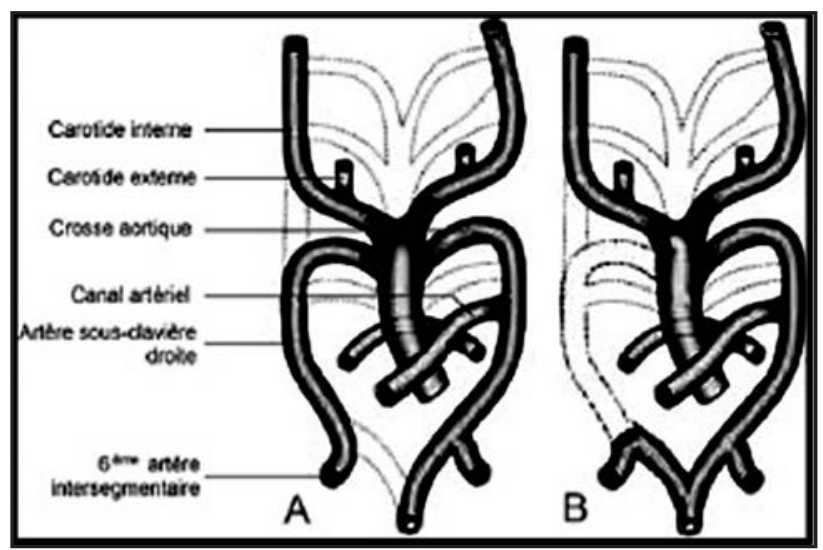

Figura 3. $(A, B)$ origen embriológico de la $\mathrm{AL}$. 
atresia pulmonar en pocos $\operatorname{casos}^{15-17}$. La AL puede también formar parte de otras enfermedades (síndrome de Di George, agenesia renal, síndrome de VACTERL) ${ }^{14,18}$.

Es casi siempre asintomática y de descubrimiento fortuito en $95 \%{ }^{19}$, sin embargo la sintomatología habitualmente encontrada -en los casos sintomáticos- es en relación con la compresión esotraqueal traducida principalmente por la disnea y la disfagia lusoria que puede resultar de todas las anormalidades del arco aórtico ${ }^{17,20}$. Sin embargo y en la edad pediátrica, predominan los signos respiratorios como la disnea, el estridor, la tos crónica, los episodios de cianosis, las infecciones bronquiales ${ }^{19}$. También, la AL puede estar sintomática en caso de la existencia de un aneurisma y en adultos que presentan ateroesclerosis 0 displasia arterial fibromuscular ${ }^{1,19}$.

Nuestra observación se caracteriza por la asociación inhabitual de una AL con otitis media a

\section{BIBLIOGRAFIA}

1. P.O Myers PO, Fasel Jhd, Kalangos A, Gailloud P. Arteria lusoria: Developmental anatomy, clinical, radiological and surgical aspects. Ann Cardiol Angeiol (Paris) 2010; 59: 147-54.

2. FALTA AUTOR. Variación retroesofágica del arco aórtico. Int J Morphol 2008; (2): 337-43.

3. Hunauld F. Examen de quelques parties d'un singe. Hist Acad Roy Sci 1735; 2: 516-23.

4. BAYFoRd D. An account of a singular case of obstructed deglutition. Memoirs Med Soc London 1794; 2: 275-86.

5. Gross RE. Surgical treatment for dysphagia lusoria. Am Surg 1946; 124: 532-5.

6. Gaujoux S, Goasguen N, de Chaisemartin C, GodirisPetit G, Munoz-Bongrand et al. Arteria lusoria et diverticule de l'oesophage. J Chir 2007; 144: 267-8.

7. Kommerell BF. Verlagerung des Oesophagus durch eine abnorm verlaufende Arteria subclavia dextra (Arteria lusoria). Forstschr Geb Roetgenstr 1936; 54: 590-5.

8. Jalal H, et al. Dysphagia lusoria: Report ofa series of six cases. Clin Res Hepatol Gastroenterol repetición causada por un reflujo en un niño sano. Generalmente la evolución natural es favorable, motivando una abstención terapéutica. La intervención quirúrgica es indicada solo en los casos con riesgo hemorrágico (aneurisma) o cuando se asocia una patología adyacente ${ }^{9}$.

\section{CONCLUSIÓN}

La AL es una malformación vascular relativamente rara y latente. En la edad pediátrica y cuando es sintomática, los signos predominantes son respiratorios y/0 esofágicos pero algunas veces se manifiesta con sintomatología atípica como fue en el caso presentado. La exploración radiológica contribuye enormemente en el diagnóstico. El crecimiento natural de la rigidez del eje esófagotraqueal permite una evolución favorable. La cirugía es reservada para los casos complicados.

(2013), http://dx.doi.org/10.1016/j.clinre. 2013.02.015.

9. H Jalaie M, J. Grommes A. Sailer and coll. Treatment of Symptomatic Aberrant Subclavian Arteries. European Journal of Vascular and Endovascular Surgery Volume 48, Issue 5, November 2014, Pages 521-526.

10. JeAnnon F. Arteria Iusoria. Étude morphodensitométrique de 150 cas. Applications cliniques. Nancy: Faculté de médecine de Nancy; 2011.

11. Stone WM, Ricotta JJ, Fowl RJ, Garg N, Bower TC, Money SR. Contemporary management of aberrant right subclavian arteries. Ann Vasc Surg 2011; 25: 508-14.

12. Abraham V, Mathew A, Cherian V and all. Aberrant subclavian artery: anatomical curiosity or clinical entity. Int J Surg 2009; 7: 106-9.

13. Myriam González-Sánchez, José Luis PardalRefoyo, Aurora Martín-Sánchez. Arteria subclavia derecha aberrante y disfagia Lusoria. Acta Otorrinolaringológica Española, Volume 64, Issue 3, Pages 244-245 (2013).

14. Evans PR. Cardiac anomalies in mongolism. $\mathrm{Br}$ Heart J 1950; 12: 258-62. 
15. CARLES D, et AL. L'artère sous-clavière droite aberrante (arteria lusoria) et le risque de trisomie 21. Analyse rétrospective de 11479 examens fœtopathologies. J Gynecol Obstet Biol Reprod (Paris) (2013), http:// dx.doi.org/10.1016/j.jgyn. 2013.10.001.

16. Zingarelli A, et al. Aberrant Right Subclavian Artery (Arteria Lusoria) Challenging 4-French Homolateral Transradial Coronary Catheterisation in Adulthood. Heart, Lung and Circulation (2015), http://dx.doi.org/ 10.1016/j.hlc.2015.04.172.

17. Myers, PA. L'Arc Aortique: embryologie, anatomie \& variantes anatomiques pour le clinicien. Université de Genève, 2009.
18. Zapata H, Edwards JE, Titus JL. Aberrant right subclavianartery with left aortic arch: associated cardiac anomalies. Pediatr Cardiol 1993; 14: $159-61$.

19. Bilel Derbel, Achref Saaidi, Refk Kasraoui, Nazih Chaouch, Fatma Aouini, Nabil Ben Romdhane, and Jamelleddine ManaA. Ann Vasc Surg 2012; 26 : 419.e1e419.e4

20. Kouki S, Fadhel A, Landoulsı M, Boujemaa $H$, Abdallah NB. Atlas tomodensitométriques des variations anatomiques des troncs supra aortiques. Feuillets de Radiologie (2014), http:// dx.doi.org/ 10.1016/j. frad.2014.10.014. 